\title{
TMEM106B haplotypes have distinct gene expression patterns in aged brain
}

Yingxue Ren ${ }^{1}$, Marka van Blitterswijk², Mariet Allen², Minerva M. Carrasquillo², Joseph S. Reddy ${ }^{1}$, Xue Wang ${ }^{1}$, Thomas G. Beach³, Dennis W. Dickson², Nilüfer Ertekin-Taner ${ }^{2,4}$, Yan W. Asmann and Rosa Rademakers ${ }^{2 *}$

\begin{abstract}
Background: Single nucleotide polymorphisms (SNPs) inherited as one of two common haplotypes at the transmembrane protein 106B (TMEM106B) locus are associated with the risk of multiple neurodegenerative diseases, including frontotemporal lobar degeneration with pathological inclusions of TDP-43. Among the associated variants, rs3173615 (encoding p.T185S) is the only coding variant; however, non-coding variants may also contribute to disease risk. It has been reported that the risk haplotype is associated with higher levels of TMEM106B and increased levels of TMEM106B cause cytotoxicity; however, the precise mechanism through which TMEM106B haplotypes contribute to neurodegeneration is unclear.

Methods: We utilized RNA sequencing data derived from temporal cortex (TCX) and cerebellum (CER) from 312 North American Caucasian subjects neuropathologically diagnosed with Alzheimer's disease, progressive supranuclear palsy, pathological aging or normal controls to analyze transcriptome signatures associated with the risk (TT) and protective (SS) TMEM106B haplotypes. In cohorts matched for disease phenotype, we used Analysis of Variance (ANOVA) to identify differentially expressed genes and Weighted Gene Co-expression Network Analysis (WGCNA) to identify gene networks associated with the risk and protective TMEM106B haplotypes.
\end{abstract}

Results: A total of 110 TCX and 116 CER samples were included in the analyses. When comparing $T$ to SS carriers, we detected 593 differentially expressed genes in TCX and 7 in CER. Gene co-expression network analyses further showed that in both TCX and CER the SS haplotype was positively correlated with gene networks involved in synaptic transmission, whereas the $\Pi T$ haplotype was positively correlated with gene networks enriched for immune response. Gene expression patterns of 5 cell-type-specific markers revealed significantly reduced expression of the neuronal marker and relative increases in all other cell markers in $\pi$ as compared to SS carriers in TCX with a similar but non-significant trend in CER.

Conclusions: By comparing the common TMEM106B risk and protective haplotypes we identified significant and partly conserved transcriptional differences across TCX and CER and striking changes in cell-type composition, especially in TCX. These findings illustrate the profound effect of TMEM106B haplotypes on brain health and highlight the importance to better understand TMEM106B's function and dysfunction in the context of neurodegenerative diseases.

Keywords: TMEM106B, Frontotemporal dementia, Co-expression networks, Differential expression, Synaptic transmission, Immune response, Progranulin

\footnotetext{
* Correspondence: Rademakers.Rosa@mayo.edu

${ }^{2}$ Department of Neuroscience, Mayo Clinic, Jacksonville, FL, USA

Full list of author information is available at the end of the article
}

(c) The Author(s). 2018 Open Access This article is distributed under the terms of the Creative Commons Attribution 4.0 International License (http://creativecommons.org/licenses/by/4.0/), which permits unrestricted use, distribution, and reproduction in any medium, provided you give appropriate credit to the original author(s) and the source, provide a link to the Creative Commons license, and indicate if changes were made. The Creative Commons Public Domain Dedication waiver (http://creativecommons.org/publicdomain/zero/1.0/) applies to the data made available in this article, unless otherwise stated. 


\section{Background}

Given the world's aging population, neurodegenerative diseases have become a major cause of disability and death. While the disease mechanisms differ, neurodegeneration often results from the accumulation of misfolded aggregated proteins in different areas of the aging brain, and this process yields cell death and inflammatory damage in those brain regions [1]. Recent studies have revealed that two common haplotypes in transmembrane protein 106B (TMEM106B) are associated with risk of multiple neurodegenerative diseases, most notably with frontotemporal lobar degeneration with pathological inclusions of TDP-43 (FTLD-TDP) [2-4], progranulin $(G R N)$-related FTLD $[3,5]$, chromosome 9 open reading frame 72 (C9ORF72)-mediated FTLD [6, 7] and hippocampal sclerosis of aging $[8,9]$. TMEM106B haplotypes were also shown to associate with the development of cognitive impairment in amyotrophic lateral sclerosis (ALS) [10] and with the presence of TDP-43 pathology in Alzheimer's disease (AD) [11] and elderly individuals without FTLD [12]. The broad involvement of TMEM106B in neurodegenerative diseases makes it an important gene to characterize and a promising target for potential therapies.

As with most risk loci identified by genome-wide association studies (GWAS), the functional variant(s) in the TMEM106B locus associated with the reported associations remains elusive. Within the associated linkage disequilibrium (LD) block, rs3173615 is the only variant encoding an amino acid change from the more common, highly conserved, threonine (Thr185; risk allele) to a serine (Ser185; protective allele) at position 185 . In vitro, the protective (Ser185) TMEM106B isoform was consistently expressed at lower levels than the risk (Thr185) TMEM106B isoform due to an increased rate of protein degradation, possibly resulting from changes in TMEM106B glycosylation [13]. In addition to the coding variant (rs3173615) numerous non-coding variants also differentiate the TMEM106B haplotypes and at least one of these variants (rs1990620) was suggested to affected higher-order chromatin architecture at the TMEM106B locus and changes in mRNA expression [14]. Indeed, expression studies in FTLD-TDP brains have shown increased TMEM106B mRNA levels in carriers of the risk haplotype [2]. Regardless of the identity of the specific functional variant(s), these findings suggest the presence of higher levels of TMEM106B in carriers of the risk haplotype and lower levels of TMEM106B in carriers of the protective haplotype. These findings are in line with cell biological studies which showed that increased TMEM106B levels were cytotoxic and led to an increase in lysosomal size and reduced lysosomal acidification [4]. Importantly, however, the specific mechanism by which TMEM106B haplotypes and changes in its expression contribute to neurodegeneration remains unknown.
In this study, we utilized RNA sequencing data of temporal cortex (TCX) and cerebellum (CER) samples from 312 North American Caucasian subjects to identify transcriptome signatures associated with the TMEM106B haplotypes. By comparing homozygote rs3173615 TT (risk) and SS (protective) carriers, we discovered differentially expressed genes in both TCX and CER regions, and identified shared gene co-expression networks between TCX and CER through which the TMEM106B haplotypes may contribute to brain function and brain health.

\section{Materials and methods Dataset description}

We used RNA sequencing data of 268 TCX and 266 CER brain samples from 312 North American Caucasian subjects with neuropathological diagnosis of $\mathrm{AD}$, progressive supranuclear palsy (PSP), pathologic aging (PA; defined as aging in nondemented elderly humans that is associated with moderate to marked cerebral amyloid deposition in the absence of significant neurofibrillary degeneration) [15] or elderly controls (CON) without clinically-significant neurodegenerative diseases [16]. The tissue processing, RNA extraction, RNA sequencing, quality control and data normalization were previously described $[16,17]$. To differentiate individuals with the TMEM106B risk and protective haplotypes we used rs3173615 as the tagging variant. Genotypes of rs3173615 for the 312 individuals were extracted by PLINK using data generated from Illumina Omni2.5 BeadChips [17].

\section{Differential gene expression analysis}

Conditional Quantile Normalization (CQN) was previously performed on the raw gene counts to correct for GC bias and gene length differences, and to obtain similar quantile-by-quantile distributions of gene expression levels across samples [17]. Based on the bi-modal distribution of the CQN normalized and log2-transformed reads per kb per million (RPKM) gene expression values, for both TCX and CER samples, protein-coding genes with average $\log 2 \mathrm{RPKM}>=-1$ in at least one haplotype group were considered expressed above detection threshold and were included in further analysis. Using this selection threshold, 16,868 genes were included for the TCX analysis and 14,994 were included for the CER analysis.

Differential gene expression analyses were performed using Partek Genomics Suite (Partek Inc., St. Louis, MO). Gene expression between the rs3173615 SS and TT individuals were compared using Analyses of Variance models (ANOVA), while correcting for RNA integrity number (RIN), age at death, sex and disease type. The selection of these covariates was based on the 
source of variation analyses from which factors with mean $\mathrm{F}$ ratio $>1.25$ were considered confounding factors that needed to be adjusted for. The Benjamini-Hochberg procedure was performed to adjust for multiple testing and control false discovery rate (FDR). Differentially expressed genes (DEG) were defined by thresholds of |fold change $\mid(\mathrm{FC})>=1.5$ and adjusted $p$ value $<0.05$. The significance of DEG overlap between TCX and CER was tested by calculating empirical $p$-value based on 100,000 simulations. Pathway analyses of differentially expressed genes were performed using MetaCore pathway analysis (Thomson Reuters) (Version 6.25).

\section{Weighted gene co-expression network analysis}

To identify groups of genes that are correlated with the TMEM106B haplotype, we performed Weighted Gene Co-expression Network Analysis (WGCNA) [18] using residual expression values calculated from adjusting for RIN, age at death, sex, and disease type. Separate WGCNA analyses were performed for the TCX and CER datasets. Signed hybrid co-expression networks were built for both WGCNA analyses. For each set of genes, a pairwise correlation matrix was computed and an adjacency matrix was calculated by raising the correlation matrix to a power. Based on the relationships between power and scale independence, the power of 4 was chosen for the TCX dataset, and the power of 14 was chosen for CER dataset. We used hybrid dynamic tree cutting, a minimum module size of 40 genes, and a minimum height for merging modules at 0.25 for both TCX and CER. Each module was summarized by the first principal component of the scaled (standardized) module expression profiles (module eigengene). For each module, the module membership measure (MM) was defined as the correlation between gene-expression values and the module eigengene. Hub genes from relevant modules, which have the highest connectivity to other genes within the module, were selected using the WGCNA function "chooseTopHubInEachModule". Each module was assigned a unique color identifier, and genes that did not fulfil these criteria for any of the modules were assigned to the gray module. To assess the correlation of modules to the TMEM106B protective (SS) haplotype, we defined the TT genotype as 0 , and SS as 1 . Modules significantly associated with TT or SS were annotated using WGCNA R function GOenrichmentAnalysis. Modules were also tested for enrichment of the respective DEG signatures using the anRichment $R$ package.

\section{Results}

Selection of study population and basic characteristics In our available dataset, we identified 77 individuals homozygous for the $\mathrm{C}$ allele at rs3173615 corresponding to Thr185 (16 AD, 29 PSP, 8 PA and 24 CON; further referred to as TT) and 65 individuals homozygous for the $G$ allele at rs3171615 corresponding to Ser185 (26 AD, 16 PSP, $5 \mathrm{PA}$ and $18 \mathrm{CON}$, further referred to as SS) in the TCX. In the CER dataset, 80 individuals were TT (21 AD, 29 PSP, 5 PA and $25 \mathrm{CON}$ ) and 64 individuals were SS (26 AD, 16 PSP, 6 PA and $16 \mathrm{CON}$ ). Since our cohort comprised samples from cases with different neurodegenerative disease as well as neuropathologically normal individuals, we further matched TT and SS study groups on pathological diagnosis and subsequently on sex and age at death, where possible. In sum, we included 55 TT and 55 SS TCX samples $(n=110)$ with equal numbers of $\mathrm{AD}, \mathrm{PSP}, \mathrm{PA}$ and $\mathrm{CON}$ in each group (Additional file 1: Table S1). In the CER, we selected $58 \mathrm{TT}$ and 58 SS samples $(n=116)$ with equal numbers of $\mathrm{AD}, \mathrm{PSP}, \mathrm{PA}$ and $\mathrm{CON}$ in each group. The 110 TCX and 116 CER samples were from 133 individual subjects (93 individuals had both CER and TCX samples). The general characteristics of the TT and SS study groups included in the analyses are presented in Table 1. TT and SS individuals were of comparable composition in terms of sex and age at death (by study design) and postmortem interval (PMI), and were also found to be similar in terms of Braak stage and brain weight.

\section{Transcriptional differences between TMEM106B TT and SS carriers in TCX and CER}

We first compared transcriptome-wide gene expression between TT and SS carriers. In the TCX dataset, we identified 593 DEGs between TT and SS carriers (Additional file 1: Table S2). In the CER dataset, we only identified 7 DEGs (Additional file 1: Table S3), none of which overlapped with the TCX DEGs. The large difference between TCX and CER suggests that the impact of TMEM106B is more prominent in a brain region affected by neurodegeneration. To further study potential similarities in the expression changes in CER and TCX we compared the top 500 genes with $|\mathrm{FC}| \geq 1.2$ between SS and TT ranked by unadjusted $p$ value in the TCX and CER dataset, which showed significant overlap in genes $(n=28 ; p=0.0008)$ (Additional file 1: Table S4).

Table 1 Characteristics of the subjects included in this study

\begin{tabular}{llll}
\hline Traits & $\Pi$ & SS & $P$ value \\
\hline Sex (male \%) & 0.41 & 0.55 & NA \\
Age at death & $81.79 \pm 9.31$ & $81.15 \pm 9.10$ & 0.69 \\
Post-mortem interval & $4.43 \pm 5.95$ & $5.29 \pm 7.39$ & 0.53 \\
Braak stage & $3.63 \pm 1.89$ & $3.78 \pm 1.99$ & 0.72 \\
Brain weight (g) & $1116.60 \pm 133.42$ & $1144.13 \pm 158.82$ & 0.37 \\
\hline
\end{tabular}

Sex and Age at death are known for all 133 subjects; Braak stage and postmortem interval (PMI) are known for 98 subjects and brain weight is known for 93 subjects 
Most of these genes are involved in neurotransmitter formation and regulation and interestingly GAD1 $\left(\mathrm{FC}_{\mathrm{CER}} 1.44 ; \mathrm{FC}_{\mathrm{TCX}} 1.90\right), \mathrm{GAD} 2\left(\mathrm{FC}_{\mathrm{CER}} 1.49 ; \mathrm{FC}_{\mathrm{TCX}}\right.$ 2.14) and SLC32A1 (FC $\mathrm{FCER}_{\mathrm{C}} 1.39$; $\left.\mathrm{FC}_{\mathrm{TCX}} 2.25\right)$, all critical for GABAergic neurotransmission, are among the shared genes.

Next we performed pathway enrichment analysis on the DEGs. In TCX, 9 out of the 10 top enriched gene ontology (GO) terms were related to synaptic signaling or cell communication (Table 2). In the CER, since only 7 genes passed the thresholds of $|\mathrm{FC}|>=1.5$ and adjusted $p$ values $<0.05$, we loosened the threshold to $|\mathrm{FC}|>=1.2$ and unadjusted $\mathrm{p}$ values $<0.05$ to allow the study of sufficient genes in the pathway analysis. In CER, we found most top enriched GO processes to be related to immune response; however, signaling was also among the top enriched GOs (Table 3).

\section{Co-expression network analyses reveals gene clusters significantly correlated with TMEM106B TT and SS carrier status in TCX and CER}

To gain further insight into the gene networks associated with the TMEM106B T (risk) and S (protection) haplotypes, we applied a gene co-expression network approach, WGCNA, which can functionally probe transcriptomic patterns of change. WGCNA is a computational tool that clusters genes in an unsupervised manner based on their correlated co-expression, and thus defines biologically relevant groups of genes that typically correspond to specific processes. WGCNA analysis of the TCX dataset identified 25 gene clusters (modules). The TMEM106B haplotype was significantly correlated with 10 modules: 4 positively correlated with SS and 6 positively correlated with TT (Table 4, Fig. 1a). WGCNA analysis of the CER dataset identified 14 modules. The TMEM106B haplotype was significantly correlated with 2 modules: 1 positively correlated with SS and 1 positively correlated with TT (Table 5, Fig. 1b). GO enrichment analysis on the modules significantly correlated with the TMEM106B haplotypes showed that synaptic transmission was a highly enriched GO in both TCX and CER from modules significantly positively correlated with the SS haplotype (modules turquoise in TCX and tan in CER). In addition, immune response was a highly enriched GO from modules significantly negatively correlated with the SS haplotype (positively correlated with TT) (modules darkred in TCX and purple in CER). This suggests that the TMEM106B haplotypes may influence brain function in multiple brain regions through similar mechanisms. Additionally, the TCX and CER synaptic transmission modules and the CER immune response module were significantly enriched for their respective DEGs, which demonstrated the consistency between the two analytic approaches (Additional file 1: Table S5).

We next identified the intramodular hub genes in the modules significantly correlated with the TMEM106B haplotype status. Hub genes are the genes with the highest connectivity to other genes of the same module; therefore, they often have important roles in the network and biological functions even if they do not meet the DEG threshold. Using the WGCNA function "chooseTopHubInEachModule", we identified one hub gene for each significant module (Tables 5 and 6). The expression level of each hub gene in SS and TT carriers is shown in Fig. 2. For the synaptic transmission modules and immune response modules in TCX and CER, we also visualized the connection among hub genes and the top 30 genes with the highest connectivity within the respective modules using VisANT [19] (Fig. 3).

\section{Cell type composition may underlie expression differences observed in TMEM106B TT and SS carriers}

The central nervous system (CNS) includes 5 main cell types: neurons, astrocytes, microglia, oligodendrocytes, and endothelial cells. We hypothesized that the relative abundance of different cell types may at least partially

Table 2 Top 10 enriched GO terms from differentially expressed genes in TCX

\begin{tabular}{llll}
\hline GO Process & $P$ value & FDR & \# Genes \\
\hline single organism signaling & $1.801 \mathrm{E}-20$ & $5.424 \mathrm{E}-17$ & 253 \\
signaling & $1.986 \mathrm{E}-20$ & $5.424 \mathrm{E}-17$ & $1.008 \mathrm{E}-16$ \\
cell communication & $5.539 \mathrm{E}-20$ & $2.908 \mathrm{E}-16$ & 253 \\
G-protein coupled receptor signaling pathway & $3.527 \mathrm{E}-19$ & $2.908 \mathrm{E}-16$ & 74 \\
chemical synaptic transmission & $4.260 \mathrm{E}-19$ & $2.908 \mathrm{E}-16$ & 59 \\
trans-synaptic signaling & $4.260 \mathrm{E}-19$ & $2.908 \mathrm{E}-16$ & 59 \\
synaptic signaling & $4.260 \mathrm{E}-19$ & $2.908 \mathrm{E}-16$ & 59 \\
anterograde trans-synaptic signaling & $4.260 \mathrm{E}-19$ & $2.366 \mathrm{E}-15$ & 59 \\
multicellular organismal process & $3.899 \mathrm{E}-18$ & $1.907 \mathrm{E}-14$ & 297 \\
cell-cell signaling & $3.492 \mathrm{E}-17$ & & 91 \\
\hline
\end{tabular}


Table 3 Top 10 enriched GO terms from differentially expressed genes in CER

\begin{tabular}{llll}
\hline GO Process & P value & FDR & \# Genes \\
\hline immune system process & $1.990 \mathrm{E}-19$ & $1.005 \mathrm{E}-15$ & 101 \\
defense response & $3.083 \mathrm{E}-17$ & $7.783 \mathrm{E}-14$ & 69 \\
regulation of multicellular organismal process & $1.448 \mathrm{E}-16$ & $2.438 \mathrm{E}-13$ & $5.996 \mathrm{E}-13$ \\
immune response & $4.750 \mathrm{E}-16$ & $1.591 \mathrm{E}-12$ & 61 \\
inflammatory response & $1.576 \mathrm{E}-15$ & $5.822 \mathrm{E}-12$ & 38 \\
response to stimulus & $6.918 \mathrm{E}-15$ & $3.187 \mathrm{E}-11$ & 224 \\
response to lipid & $4.419 \mathrm{E}-14$ & $7.520 \mathrm{E}-11$ & 61 \\
single organism signaling & $1.247 \mathrm{E}-13$ & $7.520 \mathrm{E}-11$ & 163 \\
signaling & $1.341 \mathrm{E}-13$ & $2.548 \mathrm{E}-10$ & 163 \\
positive regulation of multicellular & $5.047 \mathrm{E}-13$ & & 76 \\
Organismal process & & & \\
\hline
\end{tabular}

explain the differences in the transcriptional signatures observed in TT and SS carriers. Because the cell type composition can be estimated by cell-type-specific gene expression levels, we adopted the approached described in Allen et al. [17] and used 5 genes to estimate the cell type composition in the brain tissue samples: ENO2 for neurons (ENSG00000111674), GFAP for astrocytes (ENSG00000131095), CD68 for microglia (ENSG00000129226), OLIG2 for oligodendrocytes (ENSG00000205927), and CD34 for endothelial cells (ENSG00000174059). When we compared the expression of these 5 genes in the TT and SS carriers we found, in TCX, that the SS carriers had significantly higher neuronal gene expression and lower astrocyte, endothelium and oligodendrocyte gene expression as compared to the TT carriers. The expression of the microglial marker CD68 was also lower in SS as compared to TT carriers, but this difference was not significant. In CER, the same trends were observed, although none of the differences were significant (Fig. 4).

\section{Discussion}

In this study, we used RNA sequencing data of two brain regions to investigate the involvement of the TMEM106B risk and protective haplotypes in brain health. We identified large transcriptional differences between the protective SS and risk TT haplotypes in TCX, and much smaller differences in CER, consistent with the higher level of tissue damage and neuronal loss in the TCX region. Importantly, even though not statistically significant in CER, our data suggested similarities in the effects of TMEM106B haplotypes on the transcriptional signatures in both brain regions. By comparing the top 500 differentially expressed genes in TCX and CER with |fold change $\mid \geq 1.2$ between SS and TT, we found 28 overlapping genes, all with a fold-change in the same direction (25 are increased and 3 are decreased in SS carriers as compared to TT carriers). Some of the biggest increases in SS carriers were seen in GAD1 and GAD2, glutamic acid decarboxylases which are responsible for catalyzing the production of gamma-aminobutyric acid (GABA) from glutamate, and

Table 4 Modules significantly correlated to the TMEM106B rs3173615 status in TCX

\begin{tabular}{|c|c|c|c|c|c|}
\hline Module & Correlation & P value & Top GO & FDR & Hub gene \\
\hline turquoise & 0.35 & $2 e-04$ & synaptic transmission & $6.64 \mathrm{E}-37$ & FGF14 \\
\hline greenyellow & 0.27 & 0.004 & Ribonucleoprotein complex biogenesis & 1 & OXR1 \\
\hline darkgrey & 0.24 & 0.01 & centriolar satellite & 1 & IGSF9B \\
\hline midnightblue & 0.24 & 0.01 & ribosome binding & 0.99 & TRAPPC2L \\
\hline brown & -0.37 & $7 e-05$ & cell-cell adhesion & $6.24 \mathrm{E}-07$ & PDLIM5 \\
\hline $\tan$ & -0.25 & 0.009 & cellular response to zinc ion & 0.025 & MFSD6 \\
\hline black & -0.23 & 0.01 & vasculature development & $2.01 \mathrm{E}-19$ & $A D G R L 4$ \\
\hline darkred & -0.22 & 0.02 & humoral immune response & 0.0002 & LIMK2 \\
\hline lightgreen & -0.21 & 0.03 & cytosolic ribosome & $2.98 \mathrm{E}-123$ & RPS19 \\
\hline lightyellow & -0.2 & 0.04 & extracellular matrix organization & $9.04 \mathrm{E}-14$ & DSP \\
\hline
\end{tabular}



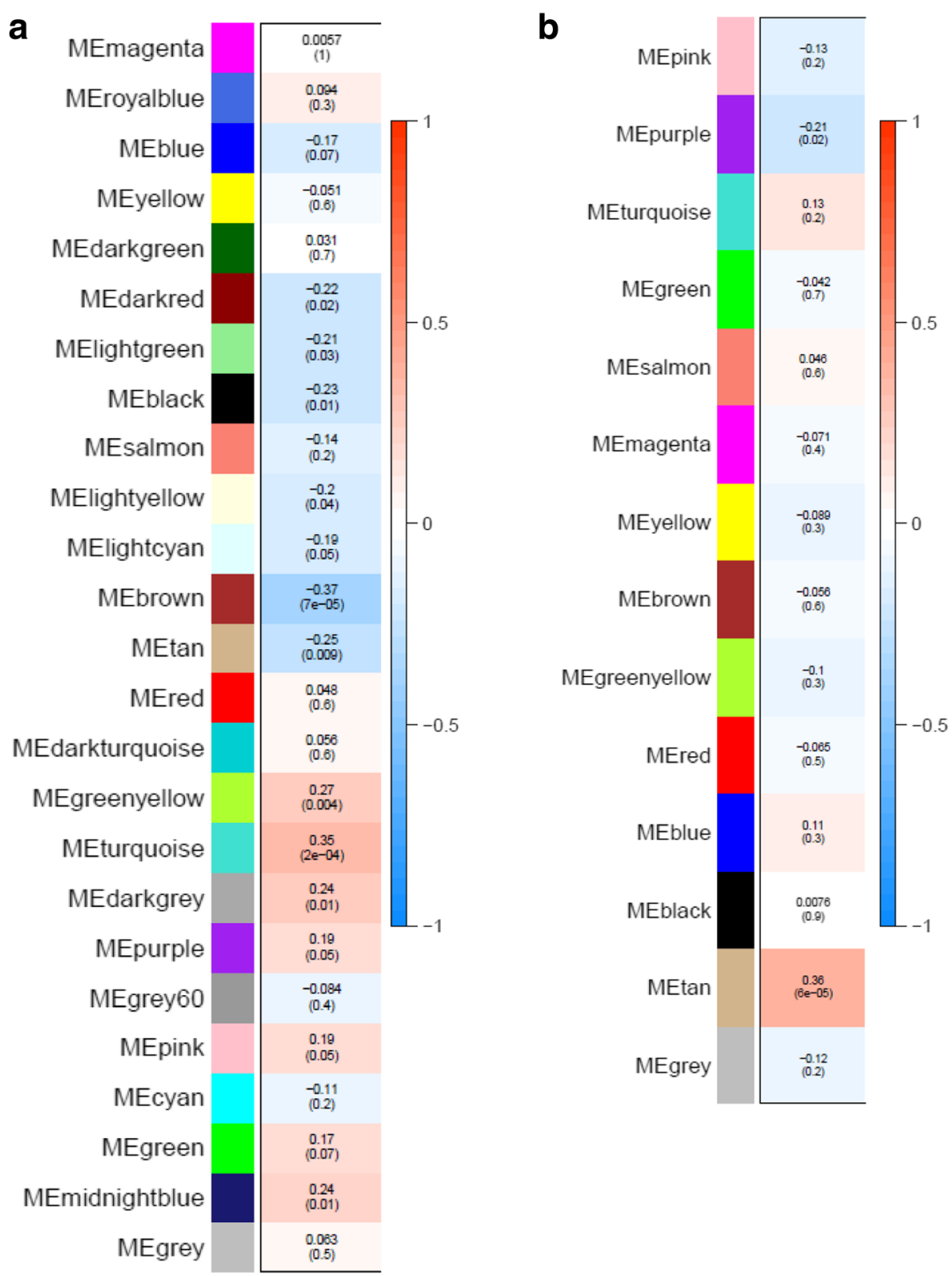

Fig. 1 Module-haplotype correlation in (a) TCX and (b) CER. Each rectangle represents a module. The top number in each rectangle is the correlation coefficient (r) between the module eigengene to haplotype, and the bottom number is the $p$ value of the correlation. The color orange represents positive correlation to the SS haplotype and blue represents negative correlation to SS (positive correlation to TT)

SLC32A1 (also known as VGAT) which is a transporter involved in the uptake of GABA and glycine into synaptic vesicles. The specific changes in GABA-related signaling are interesting in light of a recent study which reported the preferential elimination of inhibitory synapses (defined by VGAT+ immunoreactivity) in the ventral thalamus of Grn knock-out mouse brains [20]. This observation raises the possibility that more robust GABAergic signaling or an increase in the number of inhibitory synapses at baseline in SS haplotype carriers

Table 5 Modules significantly correlated to the TMEM106B rs3173615 status in CER

\begin{tabular}{llllll}
\hline Module & Correlation & P value & Top GO & FDR & Hub gene \\
\hline tan & 0.36 & $6 \mathrm{e}-05$ & synaptic transmission & $4.74 \mathrm{E}-05$ & PCP4L1 \\
purple & -0.21 & 0.02 & immune response & $1.42 \mathrm{E}-27$ & ITGB2 \\
\hline
\end{tabular}



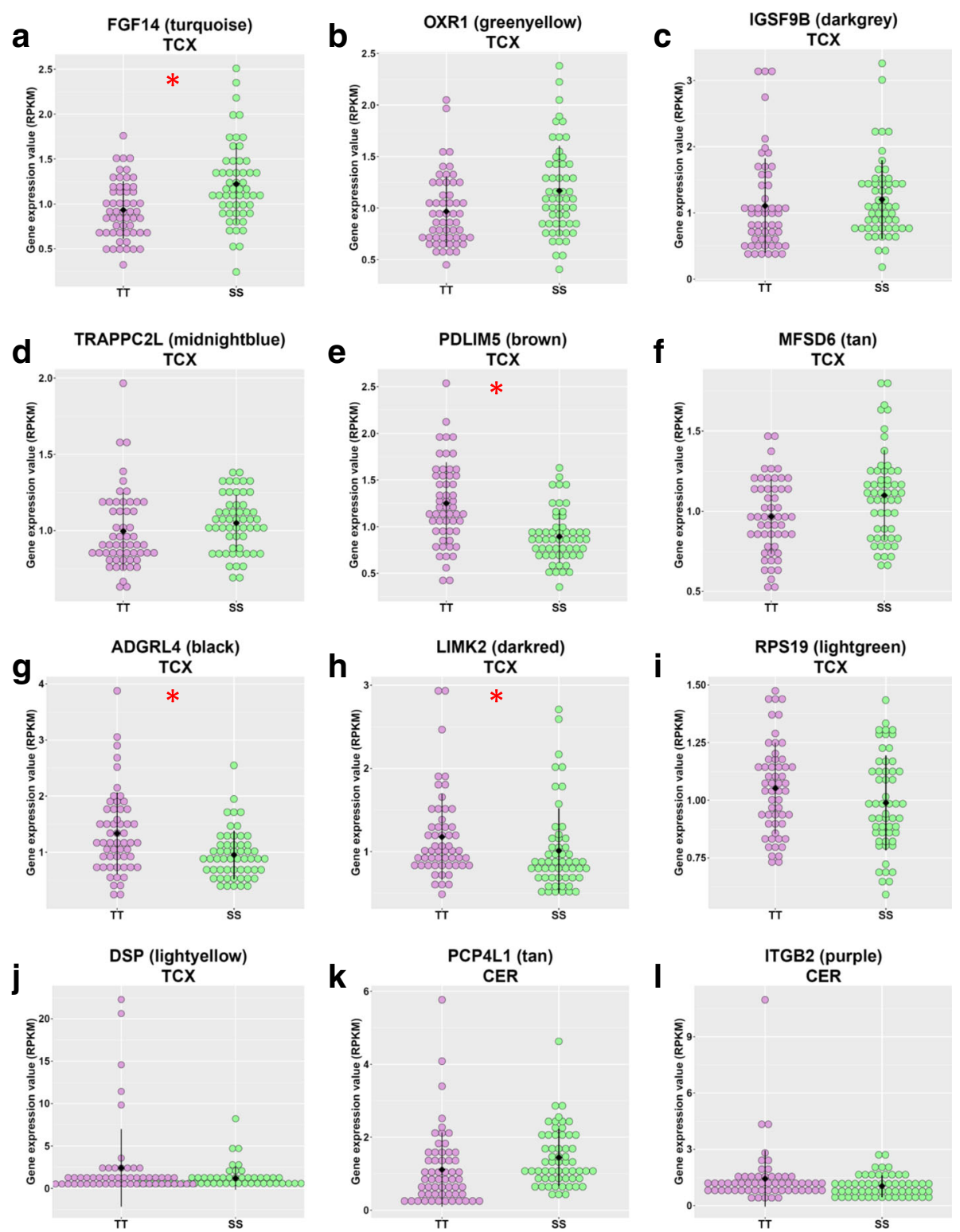

Fig. 2 Expression values of the intramodular hub genes in significant modules in TT and SS carriers in TCX (a-j) and CER (k-I). The expression values (RPKM) have been adjusted for RIN, age at death, sex and disease type. The black dots represent the mean; the black lines represent the standard deviation. Red asterisks indicate significant difference between $\Pi$ and SS (FDR <0.05)

may contribute to the profound protection conferred by this haplotype in patients with GRN mutations. Future immunohistochemical studies in human brain samples from SS and TT TMEM106B carriers are needed to confirm this hypothesis.

Using gene co-expression network analyses we further identified significant correlations of TMEM106B haplotypes with gene expression modules enriched for genes involved in similar biological processes across both brain regions. In TCX and CER, the TMEM106B SS haplotype was correlated with gene networks involved in synaptic transmission, whereas the TT haplotype was correlated with immune response networks in both brain regions, in addition to other specific gene networks such as cell-cell adhesion found only in TCX. In a further reference to the Grn knock-out mouse model, it is of interest to note that the gene expression modules most significantly correlated with the loss of Grn in cerebral cortex, cerebellum and hippocampus in mice were annotated by $\mathrm{GO}$ as involved in the innate immune response with $C 1 q a, C 1 q b, C 1 q c$, and C3 among the most connected genes in the module, similar to what we observed when we compared SS to TT carriers in the CER (purple module; Fig. 3d) [20]. Finally, using 5 genes as surrogates for 


\section{a TCX-turquoise}

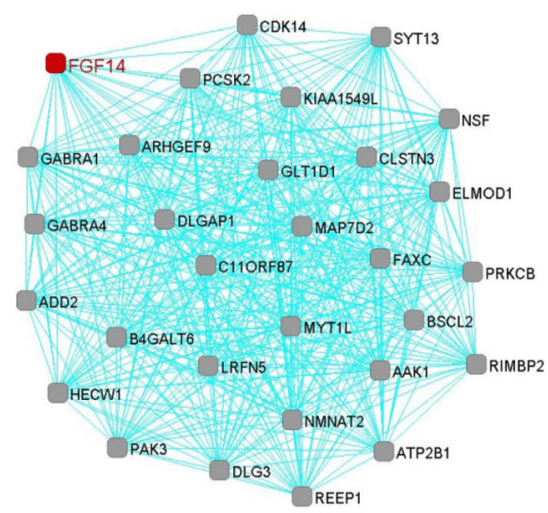

C

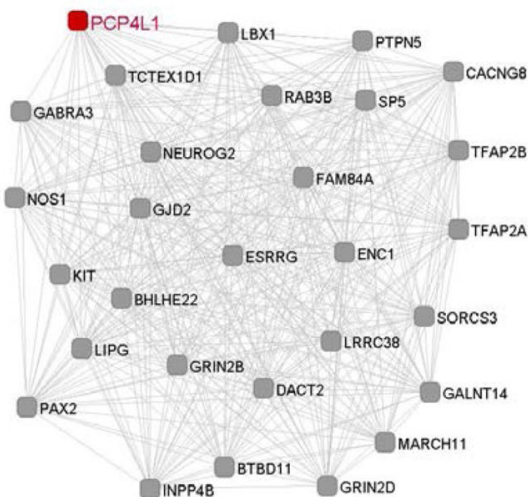

b TCX-darkred

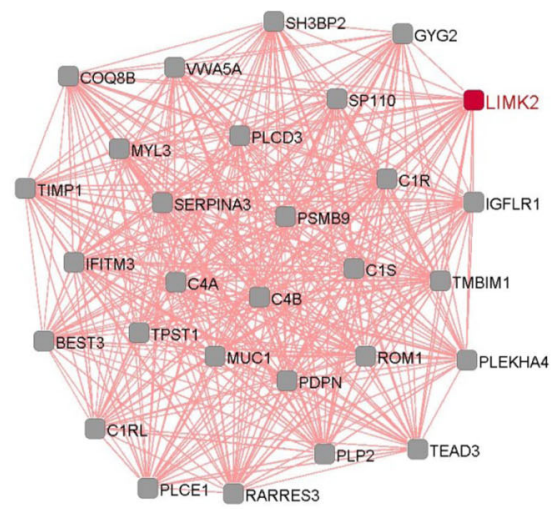

d CER-purple

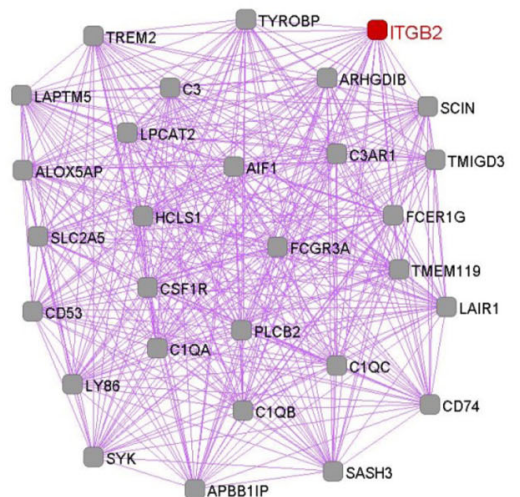

Fig. 3 Interactions of top 30 highly connected genes in the synaptic transmission and immune response modules. a and $\mathbf{b}$ are modules in TCX. c and $\mathbf{d}$ are modules in CER. The red nodes represent hub genes of each module

the 5 major brain cell types, we found that the gene networks were associated with the cell type composition in the TT and SS brains: the SS carriers showed higher neuronal gene expression as compared to the TT carriers, corresponding to an increase in networks related to synaptic transmission in SS brains; the TT carriers showed higher microglial gene expression as compared to SS carriers, in agreement with the observed enrichment for immune response networks in TT brains.

TMEM106B risk variants had previously been reported to be associated with both brain volume and connectivity. Specifically, using imaging studies, the TMEM106B risk allele was shown to significantly associate with reduced brain volume in non-demented elderly individuals, particularly in the superior temporal gyrus in the left hemisphere [21]. In addition, GRN mutation carriers with two copies of the TMEM106B risk allele demonstrated worsened brain connectivity compared to those who carried one or no risk alleles [22]. These authors found that TMEM106B haplotypes did not influence grey matter volume directly on its own, but in mutation carriers the protective TMEM106B haplotype was able to enhance the benefit of cognitive reserve on brain structure. Similarly, in our study, we found no significant differences in total brain weight between our TT and SS carriers. Instead our data suggest that the SS protective haplotype may confer higher synaptic transmission by strengthening brain connectivity in aged or diseased brain.

TMEM106B has also recently been linked to healthy neurological aging. In frontal cortex, the TMEM106B risk haplotype was associated with gene expression patterns suggestive of an older age than the individual's true chronological age [23]. Similar to our study, the TMEM106B risk haplotype was further found to be associated with increased inflammation and reduced neuronal expression in these neuropathologically normal elderly individual. Contrary to our findings, however, they did not observe an effect of TMEM106B on gene expression in cerebellar tissues. This may be related to the fact that they focused on neurologically normal individuals whereas we included a mixture of neurodegenerative 

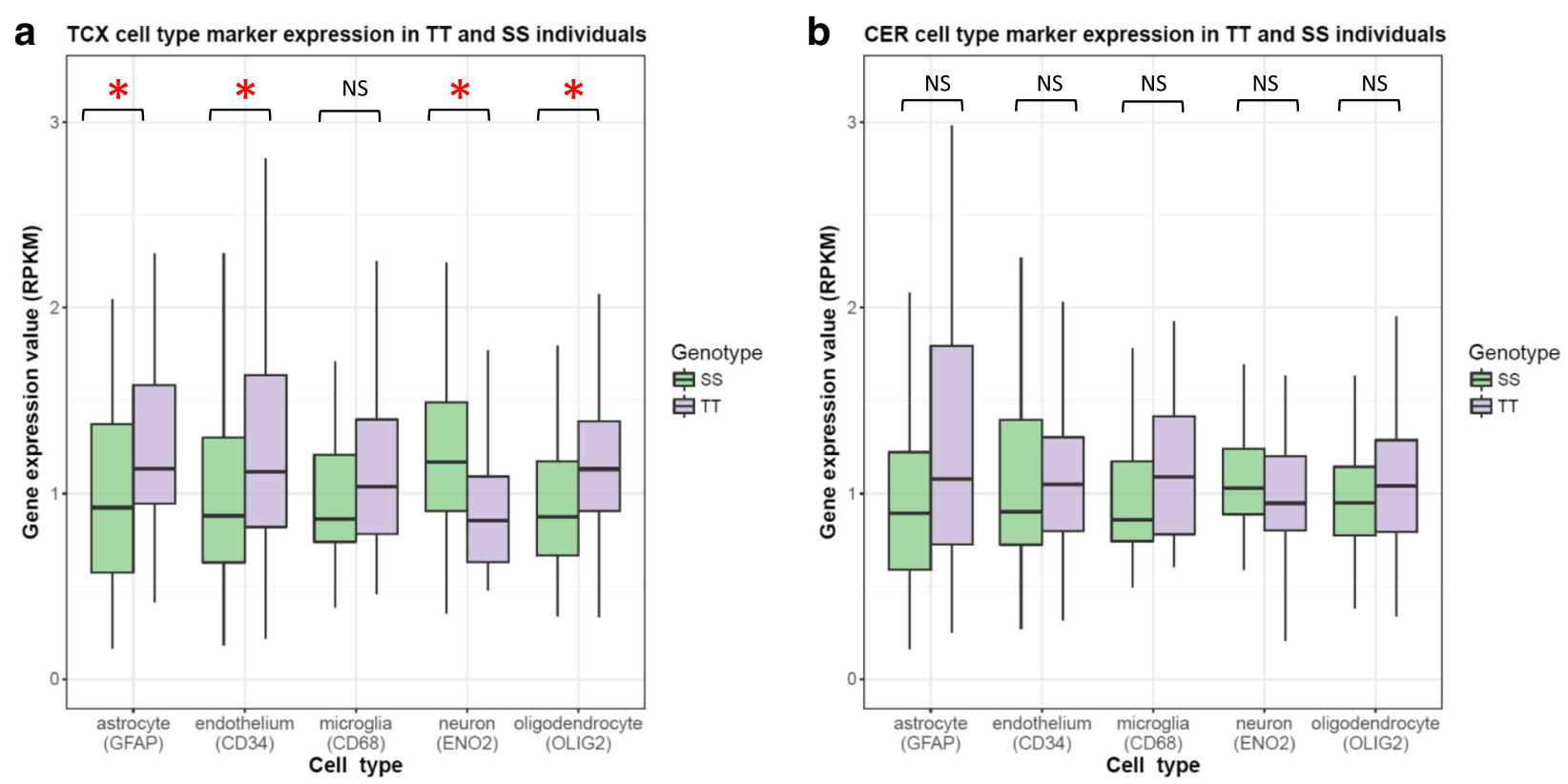

Fig. 4 Expression values (RPKM) of the 5 cell-type marker genes. The expression values (RPKM) have been adjusted for RIN, age at death, sex and disease type. a TCX samples. b CER samples. Red asterisks represent significant difference between TT and SS (FDR <0.05)

diseases and normal controls. To determine whether the significant modules we identified were mainly contributed by disease samples or controls, we performed additional WGCNA analyses with only cases in TCX $(n=74,37$ TT and 37 SS) or CER ( $n=84,42$ TT and 42 SS), and using only controls in TCX ( $n=42: 24$ TT and 18 SS) or CER ( $n=41: 25$ TT and 16 SS). In both TCX and CER, analysis of the cases identified similar modules as we did in the full TCX and CER datasets (Additional file 1: Table S6). Furthermore, independent analyses of each disease group: TCX-AD, TCX-PSP, CER-AD and CER-PSP all identified synaptic transmission modules that are significantly correlated with the TMEM106B haplotype (Additional file 1: Table S7), suggesting that the effects of the TMEM106B haplotype was not specific to disease type. Analysis of only the control samples in TCX and CER identified less modules, and synaptic transmission and immune response modules were not significantly correlated with the TMEM106B haplotype in either brain region (Additional file 1: Table S8). While the smaller sample size of the control cohorts may have reduced our power to detect significant difference, these results suggest that the effects of TMEM106B haplotypes on the transcriptome are more pronounced in disease tissues than in healthy tissues.

\section{Conclusions}

In summary, our study demonstrates significant and partly conserved effects of the TMEM106B haplotypes on the transcriptome across multiple brain regions. It is quite remarkable that a variant which is common in the normal population (minor allele frequency $=0.40$ ) can have such profound effects on gene expression and cell type composition, especially since our SS and TT study groups were matched for disease phenotypes and age at death. This suggests that additional genetic and/or environmental factors are likely to modulate the effect of the TMEM106B haplotypes on neurological and normal brain aging in individual human subjects. TMEM106B has only begun to be characterized since the discovery of its association to FTLD-TDP several years ago. While its function has mostly been linked to lysosome functions and trafficking, and recently to myelination [24], our results suggest that TMEM106B plays a broader role in the CNS response to pathological or age-related insults in multiple brain regions. Continued research into TMEM106Bs normal function and dysfunction in the context of neurodegenerative diseases will be critical and holds promise for future therapeutic strategies.

\section{Additional file}

Additional file 1: Table S1. Tissue samples available and selected for inclusion in this study. Table S2. DEGS in TCX. Positive fold change represents higher gene expression in SS than TT. Negative fold change represents lower gene expression in SS than TT. Table S3. DEGS in CER. Positive fold change represents higher gene expression in SS than $T$. Negative fold change represents lower gene expression in SS than TT. Table S4. Overlapping genes between TCX and CER based on top 500 genes with $|F C| \geq 1.2$ ranked by unadjusted $p$ value. Table S5. Enrichment of modules for their respective DEG signatures. Table S6. Significant modules identified in the TCX and CER matched cases. Table S7. Significant modules identified in separate disease groups in TCX and 
CER. Table S8. Significant modules identified in the TCX and CER controls. (DOCX $54 \mathrm{~kb}$ )

\section{Abbreviations}

AD: Alzheimer's disease; ALS: Amyotrophic lateral sclerosis; AMP-

AD: Accelerating Medicines Partnership in Alzheimer's Disease;

ANOVA: Analyses of variance; C9ORF72: Chromosome 9 open reading frame

72; CER: Cerebellum; CNS: Central nervous system; CON: Elderly controls; CQN: Conditional Quantile Normalization; DEG: Differentially expressed genes; FC: Fold change; FDR: False discovery rate; FTLD: Frontotemporal lobar degeneration; FTLD-TDP: Frontotemporal lobar degeneration with pathological inclusions of TDP-43; GO: Gene ontology; GRN: Progranulin; GWAS: Genome-wide association studies; LD: Linkage disequilibrium; MM: Module membership; PA: Pathologic aging; PMI: Postmortem interval; PSP: Progressive supranuclear palsy; RIN: RNA integrity number; RPKM: reads per kb per million; TCX: Temporal cortex; TMEM106B: Transmembrane protein 106; WGCNA: Weighted Gene Co-expression Network Analysis

\section{Acknowledgements}

The authors thank the patients and their families for their participation, without whom these studies would not have been possible.

\section{Funding}

This work was supported by the National Institute on Aging [RF AG051504 to N.E.T.; U01 AG046139 to N.E.T.], the National Institute of Neurological Disorders and Stroke [R01 NS080820 to N.E.T.; R35 NS097261-01 to R.R.] Mayo Clinic and Foundation [R.R. and Y.R.] and Mayo Clinic Center for Individualized Medicine [Y.R. and Y.W.A]. For samples collected through the Sun Health Research Institute Brain and Body Donation Program of Sun City, Arizona: The Brain and Body Donation Program is supported by the National Institute of Neurological Disorders and Stroke (U24 NS072026 National Brain and Tissue Resource for Parkinson's Disease and Related Disorders), the National Institute on Aging (P30 AG19610 Arizona Alzheimer's Disease Core Center), the Arizona Department of Health Services (contract 211002, Arizona Alzheimer's Research Center), the Arizona Biomedical Research Commission (contracts 4001, 0011, 05-901, and 1001 to the Arizona Parkinson's Disease Consortium), and the Michael J. Fox Foundation for Parkinson's Research. The funding body did not play any role in the design of the study and collection, analysis or interpretation of data and in writing the manuscript.

\section{Availability of data and materials}

The data that support the findings of this study are available through the Accelerating Medicines Partnership in Alzheimer's Disease (AMP-AD) Knowledge Portal at https://doi.org/10.7303/syn3163039 and https://doi.org/ $10.7303 /$ syn5049298. Access to the data is controlled by the institutional review board (IRB) at the Mayo Clinic.

\section{Authors' contributions}

YR, YWA and RR designed the study. YR and RR wrote the manuscript. YR and MVB performed data analysis. MA, MMC, JR, XW and NET provided intellectual input, reviewed and edited the manuscript. DWD and TGB provided tissue samples and associated data. All authors read and approved the final manuscript.

\section{Ethics approval and consent to participate}

This study uses autopsy material only and was approved by the Mayo Clinic institutional review board (IRB).

\section{Competing interests}

The authors declare that they have no competing interests.

\section{Publisher's Note}

Springer Nature remains neutral with regard to jurisdictional claims in published maps and institutional affiliations.

\section{Author details}

'Department of Health Sciences Research, Mayo Clinic, Jacksonville, FL, USA. ${ }^{2}$ Department of Neuroscience, Mayo Clinic, Jacksonville, FL, USA. ${ }^{3}$ Civin Laboratory for Neuropathology, Banner Sun Health Research Institute, Sun City, AZ, USA. ${ }^{4}$ Department of Neurology, Mayo Clinic, Jacksonville, FL, USA.
Received: 12 April 2018 Accepted: 25 June 2018

Published online: 03 July 2018

\section{References}

1. Avila J. Common mechanisms in neurodegeneration. Nat Med. 2010;16: 1372

2. Van Deerlin VM, Sleiman PMA, Martinez-Lage M, Chen-Plotkin A, Wang LS, Graff-Radford NR, et al. Common variants at 7p21 are associated with frontotemporal lobar degeneration with TDP-43 inclusions. Nat Genet. 2010; 42:234-9.

3. Finch N, Carrasquillo MM, Baker M, Rutherford NJ, Coppola G, DeJesusHernandez M, et al. TMEM106B regulates progranulin levels and the penetrance of FTLD in GRN mutation carriers. Neurology. 2011;76:467.

4. Nicholson AM, Rademakers R. What we know about TMEM106B in neurodegeneration. Acta Neuropathol. 2016;132:639-51.

5. Cruchaga C, Graff C, Chiang HH, Wang J, Hinrichs AL, Spiegel N, et al. Association of tmem106b gene polymorphism with age at onset in granulin mutation carriers and plasma granulin protein levels. Arch Neurol. 2011;68:581-6.

6. Gallagher MD, Suh E, Grossman M, Elman L, McCluskey L, Van Swieten JC, et al. TMEM106B is a genetic modifier of frontotemporal lobar degeneration with C9orf72 hexanucleotide repeat expansions. Acta Neuropathol. 2014; 127:407-18

7. van Blitterswijk M, Mullen B, Nicholson AM, Bieniek KF, Heckman MG, Baker MC, et al. TMEM106B protects C90RF72 expansion carriers against frontotemporal dementia. Acta Neuropathol. 2014;127:397-406.

8. Murray ME, Cannon A, Graff-Radford NR, Liesinger AM, Rutherford NJ, Ross $\mathrm{OA}$, et al. Differential clinicopathologic and genetic features of late-onset amnestic dementias. Acta Neuropathol. 2014;128:411-21.

9. Nelson PT, Wang WX, Partch AB, Monsell SE, Valladares $O$, Ellingson $S R$, et al. Reassessment of risk genotypes (GRN, TMEM106B, and ABCC9 variants) associated with hippocampal sclerosis of aging pathology. J Neuropathol Exp Neurol. 2015;74:75-84.

10. Vass R, Ashbridge E, Geser F, Hu WT, Grossman M, Clay-Falcone D, et al. Risk genotypes at TMEM106B are associated with cognitive impairment in amyotrophic lateral sclerosis. Acta Neuropathol. 2011;121: 373-80.

11. Rutherford NJ, Carrasquillo MM, Li M, Bisceglio G, Menke J, Josephs KA, et al. TMEM106B risk variant is implicated in the pathologic presentation of Alzheimer disease. Neurology. 2012;79:717-8.

12. Yu L, De Jager PL, Yang J, Trojanowski JQ, Bennett DA, Schneider JA. The TMEM106B locus and TDP-43 pathology in older persons without FTLD. Neurology. 2015;84:927.

13. Nicholson AM, Finch NA, Wojtas A, Baker MC, Perkerson RB, CastanedesCasey M, et al. TMEM106B p.T185S regulates TMEM106B protein levels: implications for frontotemporal dementia. J Neurochem. 2013;126:781-91.

14. Gallagher MD, Posavi M, Huang P, Unger TL, Berlyand Y, Gruenewald AL, et al. A dementia-associated risk variant near TMEM106B alters chromatin architecture and gene expression. Am J Hum Genet. 2017;101:643-63.

15. Dickson DW, Crystal HA, Mattiace LA, Masur DM, Blau AD, Davies P, et al. Identification of normal and pathological aging in prospectively studied nondemented elderly humans. Neurobiol Aging. 1992;13:179-89.

16. Allen M, Carrasquillo MM, Funk C, Heavner BD, Zou F, Younkin CS, et al. Human whole genome genotype and transcriptome data for Alzheimer's and other neurodegenerative diseases. Scientific Data. 2016;3:160089.

17. Allen M, Wang X, Burgess JD, Watzlawik J, Serie DJ, Younkin CS, et al. Conserved brain myelination networks are altered in Alzheimer's and other neurodegenerative diseases. Alzheimers Dement. 2018;14:352-66.

18. Langfelder $P$, Horvath S. WGCNA: an R package for weighted correlation network analysis. BMC Bioinformatics. 2008;9:559.

19. Hu Z, Chang YC, Wang Y, Huang CL, Liu Y, Tian F, et al. VisANT 4.0: integrative network platform to connect genes, drugs, diseases and therapies. Nucleic Acids Res. 2013;41:W225-31.

20. Lui H, Zhang J, Makinson SR, Cahill MK, Kelley KW, Huang HY, et al. Progranulin deficiency promotes circuit-specific synaptic pruning by microglia via complement activation. Cell. 2016;165:921-35.

21. Adams $H H H$, Verhaaren BF, Vrooman HA, Uitterlinden AG, Hofman A, van Duijn CM, et al. TMEM106B influences volume of left-sided temporal lobe and interhemispheric structures in the general population. Biol Psychiatry. 2014;76:503-8. 
22. Premi E, Formenti A, Gazzina S, Archetti S, Gasparotti R, Padovani A, et al. Effect of TMEM106B polymorphism on functional network connectivity in asymptomatic GRN mutation carriers. JAMA Neurology. 2014;71:216-21.

23. Rhinn $\mathrm{H}$, Abeliovich A. Differential aging analysis in human cerebral cortex identifies variants in TMEM106B and GRN that regulate aging phenotypes. Cell Systems. 2017:4:404-15.

24. Simons C, Dyment D, Bent SJ, Crawford J, D'Hooghe M, Kohlschütter A, et al. A recurrent de novo mutation in TMEM106B causes hypomyelinating leukodystrophy. Brain. 2017;140:3105-11.

- fast, convenient online submission

- thorough peer review by experienced researchers in your field

- rapid publication on acceptance

- support for research data, including large and complex data types

- gold Open Access which fosters wider collaboration and increased citations

- maximum visibility for your research: over $100 \mathrm{M}$ website views per year 